\title{
openheart Open Heart - The new BMJ cardiovascular journal, advocating open access, open peer-review and open data
}

\author{
Pascal Meier, ${ }^{1,2}$ Georg M Fröhlich, ${ }^{1}$ Guido Knapp, ${ }^{3}$ Saidi Mohiddin, ${ }^{4}$ \\ Ben Goldacre, ${ }^{5}$ Duolao Wang, ${ }^{5}$ Christoph Kustosz, ${ }^{3}$ James J DiNicolantonio, ${ }^{6}$ \\ Shah Ebrahim, ${ }^{5}$ Andreas Baumbach, ${ }^{7}$ Niels van Royen, ${ }^{8}$ Juan-P Casas ${ }^{5}$
}

To cite: Meier $P$,

Fröhlich GM, Knapp G, et al. Open Heart - The new BMJ cardiovascular journal, advocating open access, open peer-review and open data. Open Heart 2013;1: e000007. doi:10.1136/ openhrt-2013-000007
${ }^{1}$ Cardiology, University College London Hospital, London, UK

${ }^{2}$ Yale Medical School, New Haven, CT, USA

${ }^{3}$ Statistics, TU Technical University Dortmund, Dortmund, Germany ${ }^{4}$ Cardiology, Barts and The London NHS Trust, London, UK

${ }^{5}$ London School of Hygiene \& Tropical Medicine, London, UK

${ }^{6}$ Mid America Heart Insitute, Kansas City, MO, USA ${ }^{7}$ Bristol Heart Institute, University Hospital Bristol, Bristol, UK

${ }^{8}$ Cardiology, VU Medical Centre, Amsterdam, Netherlands
Open Heart is a new international cardiovascular journal from BMJ and the British Cardiovascular Society, launching in January 2014.

The journal will publish a broad range of cardiovascular articles, including research protocols/proposals, findings of translational research, observational studies, randomised trials, systematic reviews and meta-analyses in the field of cardiovascular medicine. We will also publish narrative reviews and editorials focusing on research published and research methodology.

Open Heart's editorial team will primarily assess original contribution for robust research methodology and will also publish high quality research that presents confirmatory or negative results while novelty of findings will receive a lower level of attention. In an attempt to reduce publication bias, Open Heart also considers smaller, specialist studies providing preliminary or descriptive data that might be considered 'hypothesis generating' rather than definitive.

\section{HOW IS OPEN HEART DISTINCT FROM HEART}

Heart is a 'conventional' print journal, and like similar journals, is limited in the amount of research that can be published per issue. Consequently, Heart prioritises research articles, usually based on novelty and importance. Open Heart is published exclusively online, without any page restriction, and therefore allowing us to base our assessment purely on the quality of the study. Manuscripts that have been rejected by Heart due to a lack of priority or novelty will be transferred to Open Heart, with authors' agreement and without requiring any reformatting.
Any previous peer reviewers' comments will also be considered which will substantially speed up manuscript processing time.

Further key advantages of Open Heart are:

- Worldwide accessibility - high impact

Open access ensures that everybody will be able to access the articles free of cost, anywhere. Hosting by bmj.com ensures visibility; exceptional articles will attract press release through BMJ's Press Office. Open Heart will actively promote its articles using well-read BMJ channels such as blogs, social media and through close editorial board contacts with medical journalists.

- Fast review and publication process

Unlike other journals, beyond the standard submission process, authors have the option of a fast-track model, whereby abstracts are the initial submission on which a rapid decision will be provided as to whether the paper has the potential to be accepted. This should save the time of both authors and reviewers by protecting them from investing valuable resources on manuscripts with fundamental limitations or which are outside the scope of Open Heart, and allowing prompt publication of time-sensitive articles. Following the initial decision, we will invite successful authors to submit their full text article for prompt peer and statistical review.

Fast-track model:

1. Abstract submission.

2. Prompt review by an expert editorial team with quick feedback.

3. Invitation for full submission or rejection.

4. Editor-in-Chief/assigned editor will organise the peer review process, usually involving $\geq 2$ external peer reviewers and a statistical editor/reviewer. 
5. Decision on the paper by editorial team, usually involving a statistical editor.

6. Quick online publication - normally within 10 days.

- Open peer review

Open Heart will apply an open peer review process, where the name of the peer reviewer is known to the authors and vice versa. ${ }^{1}$ This will promote a constructive and prompt review process.

Open Heart will benefit from BMJ's experience with fast turnaround times from acceptance to online publication - the average is currently just 20 days and we will continue to strive for improvements. At all times, scientific soundness has the highest priority. The journal will publish under a continuous publication model, whereby all articles are published online, fully citeable, as soon as authors have signed off on proofs. Articles will be deposited immediately with PubMed Central and included in PubMed.

\section{EXPERIENCED, CONSTRUCTIVE EDITORIAL TEAM}

The editorial team consists of an international team of experts in their fields, as well as in peer review and editing, whose experience comes from our sister journal Heart, other journals and several members are part of the Cochrane Heart Group based at the London School of Hygiene Tropical Medicine; all are highly experienced in assessing research methodology and research quality. Heart has recently started to work with the Cochrane Heart Group, ${ }^{2} 3$ and Open Heart is committed to be part of and to further intensify this collaboration. A novel aspect of Open Heart compared with other journals is the involvement of statistical editors who are an integral part of the core editorial team.

\section{OPEN DATA}

We believe that all trials must be registered, with their methods and results made publicly available (AllTrials initiative $)^{4}$. Transparency is important, to reduce duplication of effort within the scientific community, and to avoid unnecessary bias in the information made available to clinicians and patients about the risks and benefits of treatments. We also believe that the research community must move towards 'open data' - sharing the raw data analysed in a study - with due respect to the risks of de-anonymisation where individual patient data has been used. Authors will be encouraged to make their data available via a specialist data repository (such as Dryad) or an institutional repository, as supplementary files attached to the article, or upon request from readers. Fees for Dryad deposit will be sponsored by the journal. As an additional benefit, authors who agree to make their data accessible receive a discount on their Article Processing Charges (APCs).

Conflict of interest: None.

Contributorship PM has drafted the text. All acknowledged associate editors have revised it critically for its intellectual content and have agreed to the final version of the text.

Open Access This final article is available for use under the terms of the Creative Commons Attribution Non-Commercial 3.0 Licence; see http:// journals.bmj.com/site/openheart

\section{REFERENCES}

1. Groves T. Is open peer review the fairest system? Yes. BMJ 2010;341:c6424.

2. Meier P, Timmis A, Casas JP, et al. Cochrane Corner: is there a benefit of using intracoronary vasodilators in ST segment elevation myocardial infarction? Heart 2010;99:1381-2.

3. Timmis A, Meier P, Ebrahim S, et al. Heart and the Cochrane Collaboration. Heart 2010;99:1379-80.

4. Goldacre B. Are clinical trial data shared sufficiently today? No. BMJ 2013;347:f1880. 